\title{
An Optimization Method for Missile Testing Vehicle Based on Genetic Algorithm
}

\author{
LIU Ai-zhen \\ Department of Information Engineering, \\ Ordnance Engineering College, \\ Shijiazhuang, China \\ liuaizhen_163@163.com \\ GAO Xiu-feng \\ Department of Information Engineering, \\ Ordnance Engineering College, \\ Shijiazhuang, China
}

\author{
CHEN Li-yun \\ Department of Information Engineering, \\ Ordnance Engineering College, \\ Shijiazhuang, China
}

\author{
LI Xi \\ Department of Information Engineering, \\ Ordnance Engineering College, \\ Shijiazhuang, China \\ lazzlx@yahoo.com.cn
}

\begin{abstract}
Planning the optimum number of Missile Testing Vehicles is very important to decrease testing cost especially testing time of kinds of missiles. To determine the number of vehicles, the mathematics model was established and an optimization method based on Genetic Algorithm was proposed. And an encoding rule based on vary numbers of the vehicle, maximum testing time and variable task list was designed; special cross operator, special transformation operator and mutation operator all based on one chromosome were proposed. The simulation results show that the solutions of this method can get a very perfect balance between testing cost and testing time compared with the other methods, and can adapted in the demands of accurate support in wartime.
\end{abstract}

Keywords - Missile Testing Vehicle; Optimization Method;
Genetic Algorithm

\section{INTRODUCTION}

New military reform has been influencing and changing every aspects of military field profoundly. As an important content during wartime, ammunition support pattern is undergoing a process of transforming from "rough

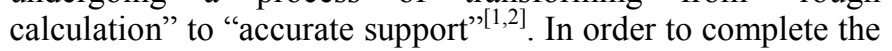
ammunition support of wartime effectively and at a low cost, according to the battlefield request, determining the quantity of equipments accurately is of great significance in ammunition supporting.

According to the process of ammunition support in future warfare, the supplying types and quantity of the ammunition that the war needs could be real-time perceived and ascertained. Among them, the ammunitions of precise-navigation weapons are generally used on key or important targets due to its high cost and low utilizing amount. To ensure the ammunition is in good condition, it must be tested before use. It means that all the missiles must be tested by the missile testing vehicle before transportation, and only after the missile is qualified could it be loaded and transported.
In this link, the quantity of the missile testing vehicle is related to many problems such as the test cost and support extent. Above all, research on the optimal deployment of missile testing vehicle is of great importance to decrease the cost and increase the effectiveness of ammunition support.

There are three types of methods to test the missiles at present: serial testing method, parallel testing method and serial-parallel testing method. Serial testing method means testing the missiles serially by only one missile testing vehicle. This kind of method use less missile testing vehicle and cost less, but the test time is too long. Parallel testing method means testing all the missiles at the same time. This kind of method cost the least time, but it need too many missile testing vehicles and cost a lot. Serial-parallel testing method means completing the test by several missile testing vehicles, each of which is assigned some missiles and all vehicles test their assigned tasks serially. The testing time is the latest time of all missile testing vehicles completed the test. This method usually uses the estimated method base on experience to decide the number of the missile testing vehicles. Since the method came from a large number of actual combats, it is rational under some conditions, but it lacks the scientific, reasonable analyses. So this method often causes the waste and insufficiency of support vehicle. If the estimated number of the testing vehicle is redundant, it not only leads to higher cost, but also leads to more danger caused by many supplying targets. However, if the estimation is inadequate, it would directly influence on the final victory of war. In order to solve the above problem of traditional method, this thesis established a mathematics model and designed special Genetic Algorithm to obtain the optimum number of the missile testing vehicles.

\section{ANALYSIS AND DESCRIPTION ON THE PROBLEM}

\section{A Problem Analysis}

The missile types of precision-guided equipments are 
different, thus the testing process, the testing time are also different. The support equipments testing missiles, missile testing vehicles, are universal. When the different kinds of missiles are being tested, the vehicle is the same, only different specific modules are used. Therefore, to shorten the overall testing time of all the missiles the rational number of missile testing vehicles and scientific testing order of kinds of missiles, is the key to reducing testing costs and testing time. Considering that the process to test the same kind of missiles is also the same, thus we could regard the testing time of the same kind of missiles are the same, too. Here, since missile in fault is rare, the case is negligible. However, when testing different types of missiles, the testing process is clearly different, and the testing time is also different. Testing time is mainly influenced by the following factors: (1) kinds and numbers of missiles. When the other deployment is the same, the more kinds and numbers of missiles are, and the longer the testing time is. (2) number of missile testing vehicle. When the other deployment is the same, the more vehicles mean the shorter the testing time of missile. (3) missile testing task sequences of missile testing vehicles. The other deployment is the same, the different missile testing sequences assigned to each missile testing vehicle mean different testing time. Rational assignment can effectively shorten the overall testing time of missiles, otherwise the overall testing time will be lengthen. Therefore, the rational quantity of missile testing vehicles and the rational missile testing sequence is the key to acquire the shortest testing time.

\section{B The Mathematical Model}

Based on the above analysis of the missile testing vehicle, the total number of various types of missiles is denoted as integer variable $M$; the number of missile testing vehicle is denoted as integer variable $C$. Since $\mathrm{C}$ and $\mathrm{M}$ are integer value, so the vehicle can be numbered with $1,2, \ldots \mathrm{C}$; the missile can be numbered with $1,2, \ldots, \mathrm{M}$. There are two cases.

- If $M \leq C$, the $C$ vehicles is enough, $M$ missiles can be tested in parallel, so this case is need not to consider the optimization problem.

- If $M>C$, the $C$ vehicles only test $C$ missiles in parallel at one time, so $M$ missiles must be tested in batches and for several times. Each missile testing vehicle must test several missiles, how to assign the missiles for these vehicles is the key. We should judge and balance comprehensively on determining the missiles testing sequence of each missile testing vehicle according to the maximum testing time affection.

Here we mainly discuss the optimum number problem of the missile testing vehicle on the later case, $M>C$.

(1) To determine the optimum testing time of the same kind of missiles. The same kind of missiles is regarded as the same testing time, denoted as time variable $t$, so the total testing time of all the same kind of $m$ missiles can be computed by formula (1).

$$
T_{\text {finish }}=\left\lceil\frac{m}{C}\right\rceil \times t
$$

In the formula (1), $m$ is not vary, $C$ can be taken different values. The testing completion time of $M$ missiles may be different, or may be the same. It depends on whether $M$ divided by $C$ up to take the integer value is the same.

If the maximum testing time constraints, $T_{\max }$, is given, the minimum or optimum number of the testing vehicles can be obtained according to the formula (2).

$$
x_{\min }=\min (C)
$$

s.t $\mathrm{T}_{\max } \geq T_{\text {finish }} \times t$

$$
T_{\text {finish }}=\left\lceil\frac{m}{C}\right\rceil \times t
$$

(2) To determine the optimum testing time of kinds kind of missiles. For different kinds of missiles, both the testing processes and the corresponding testing times are different. Assume the testing times sequence of all $M$ missiles is denoted as array $t$.

$$
t=\left[\begin{array}{llll}
t_{1} & t_{2} & \ldots & t_{M}
\end{array}\right]
$$

Assume that the maximum testing time constraints, $T_{\max }$, is given, then the objective functions and constraints calculating the optimum or minimum number of the missile testing vehicles are the following formula (3), formula (4), formula (5).

$$
x_{\min }=\min (C)
$$

s.t. $\quad \max \left(T_{1}, \ldots, T_{j}, \ldots ., T_{C}\right) \leq T_{\max }$

$$
\begin{gathered}
T_{j}=t_{j \text { finish }}-t_{j s t a r t} \\
=\sum_{\substack{i=1 \\
t_{j i} \in \text { Set }_{j}}}^{i \leq a} t_{j i} \\
\text { Set } t_{j}=\left[t_{j 1}, \ldots, t_{j i}, \ldots, t_{j a}\right] \\
j, C, a \in[1,2, \ldots] \\
a \leqslant M \\
j \leqslant C
\end{gathered}
$$

$T_{j}$---- it represents the total testing time that the testing vehicle No. $j$ finishes its testing tasks assigned. Formula (4) gives the way to calculate $T_{j}$. Formula (5) represents that $a$ missiles was assigned to the missile testing vehicle No.j to be tested.

From formula (3) and (4), it can be seen that both the number of the testing vehicles and the missile testing sequences assigned to each testing vehicle have influenced on the total testing time. In Summary, the object above can be described as to find the optimum missiles assignment scheme for all testing vehicles, in which the maximum testing time constraints can be satisfied with the least testing vehicles. 


\section{GENETIC ALGORITHM DESIGN ON THE PROBLEM}

Genetic algorithm ${ }^{[3]}(\mathrm{GA})$ is a search heuristic that mimics the process of natural evolution. This heuristic is routinely used to generate useful solutions to optimization and search problems. It has been widely and successfully applied in combinatorial optimization problem fields ${ }^{[4,5]}$. Here using it to solve combinatorial optimization problem on the missile testing vehicles. According to the testing characteristics of kinds of missiles, we specially designed an encoding method and a variety of genetic operators as following.

\section{A Encoding Strategy on Chromosome}

From above all, the testing time series of $M$ missiles is generally supposed as: $t=\left[\begin{array}{llll}t_{1} & t_{2} & \ldots & t_{M}\end{array}\right]$, and the quantity of missile testing vehicle is denoted as variable $C$. And the maximum testing time, $T_{\max }$, can be determined according to the demands of war. Considering the influence of the testing, the chromosome encoding strategy based on list method is defined as shown in Fig.1. $(\mathrm{M}=10, \mathrm{C}=3)$

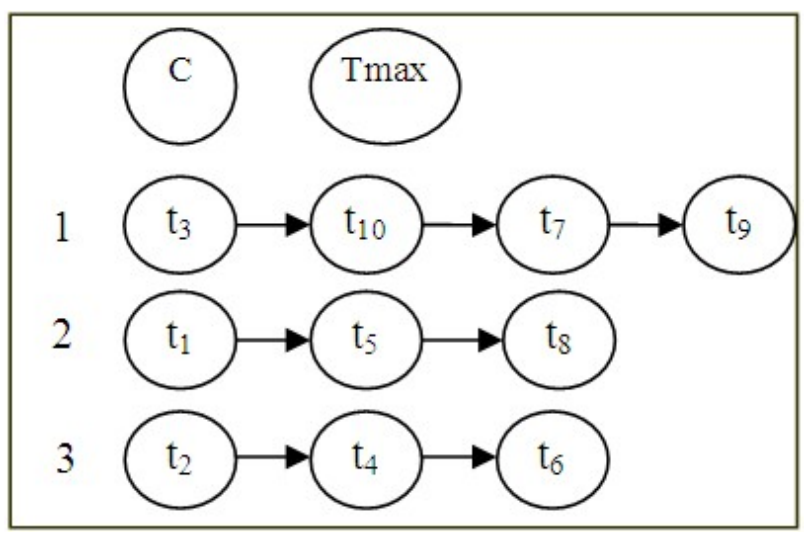

Figure 1 the encoding strategy

In the Fig.1, $C$ denotes the number of missile testing vehicles; $T_{\max }$ is the maximum testing time; 1,2 , and 3 denotes the missile testing vehicle id. And $t_{1}, t_{2}, \ldots t_{10}$ denoted as the testing time of the missiles id in suffix.

\section{B Fitness Function on Chromosome}

The fitness function is used to evaluate all the individuals of population and also is used to control genetic manipulation. Here, using GA is to find the optimum or the fewest testing vehicle quantity according to the maximum testing time, so the less fitness value of chromosome is the better. However, all selection operations in GA are trying to maximize the fitness, therefore, the fitness function of the problem (denoted as: fit (s)) is defined as:

$$
f i t(s)=M A X-T_{\text {finish }}(S)
$$

MAX - an enough constant ensuring fit $(s) \geq 0$.

$T_{\text {finish }}(S)$ - the total testing time of all the missiles.

\section{Initial Population Generation}

First the population size is denoted as variable $p$, and the missile testing time sequence is denoted as set $t=\left[t_{1}, \ldots . t_{M}\right]$, the number of missile testing vehicles is denoted as variable $C$.
The initial population can be generated according to the following steps.

(1) Set the value of $p$, and another set $t^{\prime}=t$, and $i=0$;

(2) Select the value of $C$ randomly, and set $S c=[1,2, \ldots$, C];

(3) If $t^{\prime}<>\Phi$, go to step (4); otherwise $t^{\prime}=t$;

(4) From the set $t$ ', missiles set to be tested, randomly select an element; also from set $S c$, the missile testing vehicles id, randomly select an element; And added the selected missile element to the task list of selected missile testing vehicle element; then remove the selected missile element from the set $t^{\prime}$.

(5) Repeat steps (4) until set $t$ ' is empty. This means all the missiles to be tested have been allocated, and a scheduling scheme, also called as chromosome, has been generated.

(6) $\quad i=i+1$;

(7) if $i<p$, go to step (3), otherwise, the initial population is acquired.

\section{Designing Genetic Operators}

\section{1) choosing operator}

With roulette method, the better fitness value one chromosome has, the bigger chosen-probability it is. Since the immergence of the population make the chromosome with the best fitness value could not be sure chosen, here roulette method was applied and best-individual is retained to make sure the best individual to be chosen in this algorithm.

\section{2) cross operator}

Considering of the problem limitation, new valid chromosome cannot be derived from two chromosomes by common cross operator. A special cross operator only used in single chromosome is designed. The process of cross operator is shown as Fig. 2 and Fig. 3.

(1) choose one chromosome randomly such as Fig. 2;

(2) choose two missile assignment sequences(such as 1,2 in Fig.2) from testing vehicles id randomly, and choose two cross points randomly(such as $t_{10}, t_{11}$ in Fig. 2);

(3) exchange all their assignments after two cross points, and new chromosome can be obtained shown as Fig. 3.

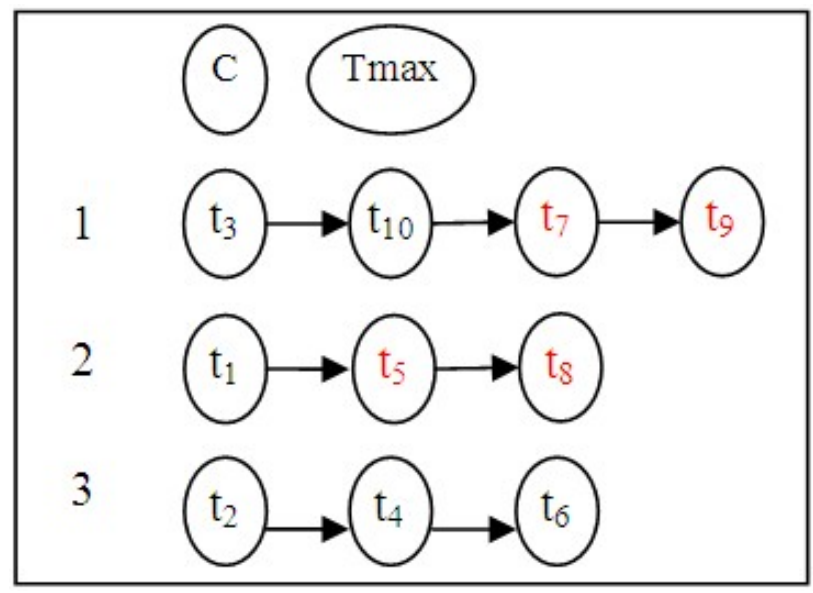

Figure 2 Chromosome before using the cross operator 


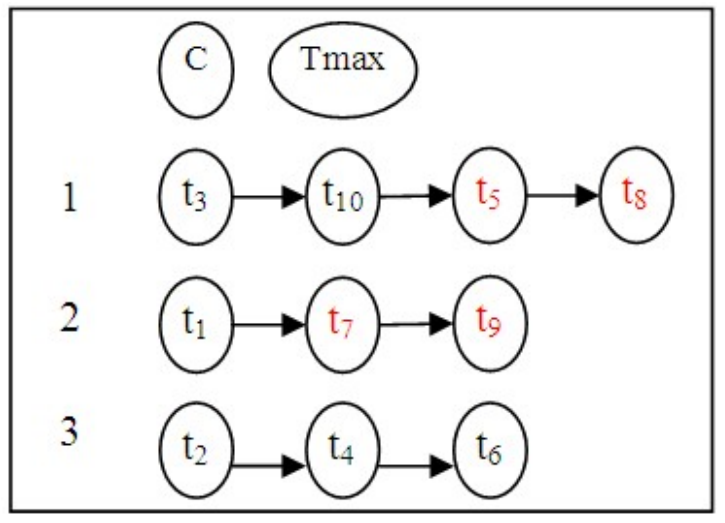

Figure 3 Chromosome after using the cross operator

\section{3) Transformation Operator}

To increase diversity of chromosomes, a special transformation operator is designed. The transformation process is shown as Fig. 4 and Fig. 5.

(1) choose one chromosome randomly, such as Fig. 4;

(2) choose one missile point from one testing vehicle id randomly, such as $t_{8}$ of vehicle id, 1 , in Fig. 4;

(3) move the missile, $t_{8}$, to another testing vehicle missiles sequence to test, such as vehicle id 3. And new chromosome derived from Fig. 4 is shown as Fig. 5.

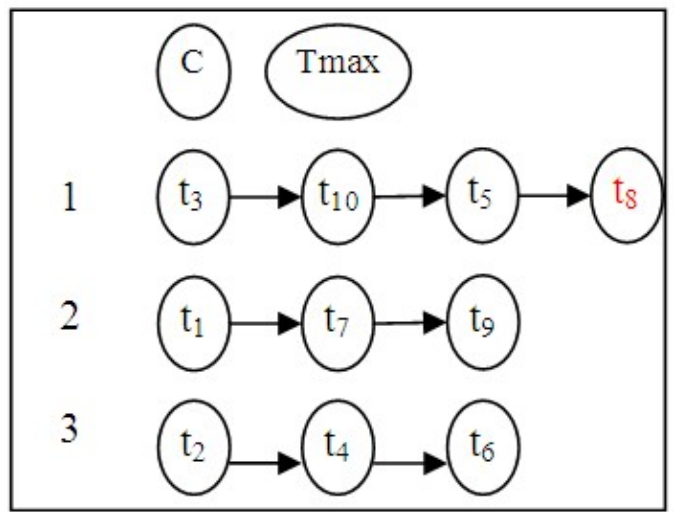

Figure 4 Chromosome before using the transformation operator

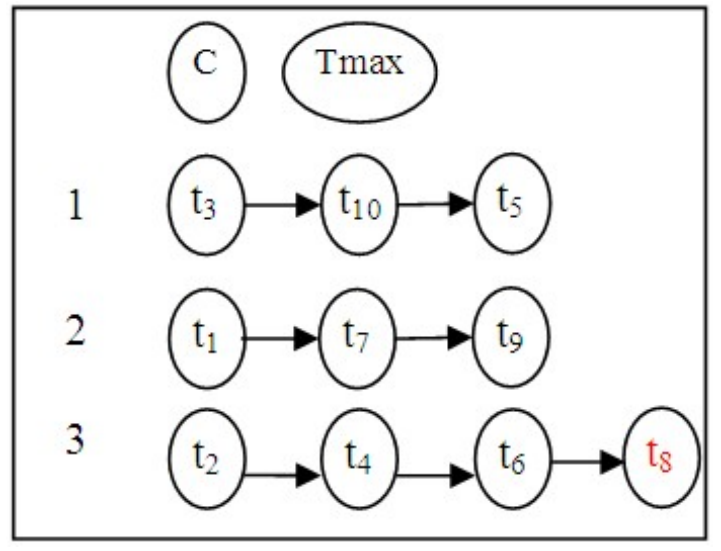

Figure 5 Chromosome after using the transformation operator
4) Mutation Operator

(1) choose one chromosome randomly, such as Fig. 6;

(2) choose separately and randomly one missile of two testing vehicles id selected by randomly, such as $t_{7}$ of vehicle id 2 and $t_{4}$ of vehicle id 3 in Fig. 6;

(3) exchange the chosen missile points. And the new chromosome applied the mutation operator is shown as Fig. 7.

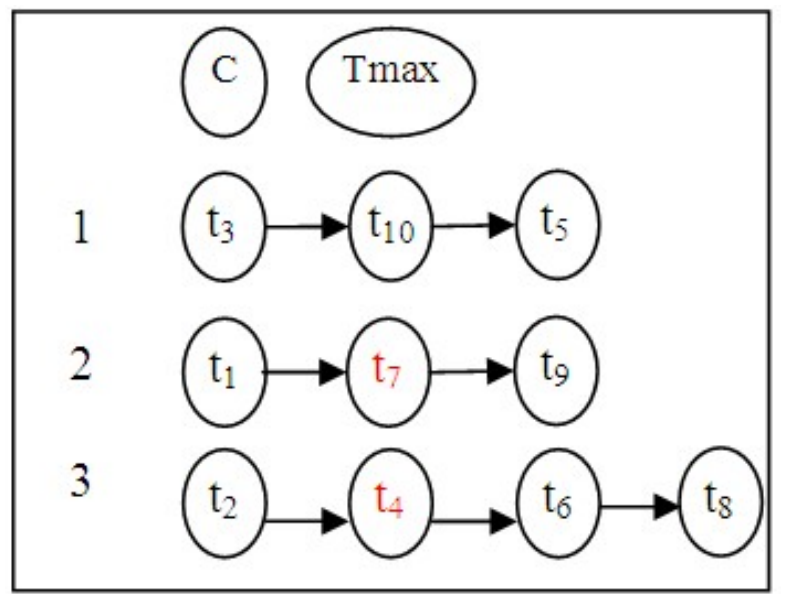

Figure 6 Chromosome before using the mutation operator

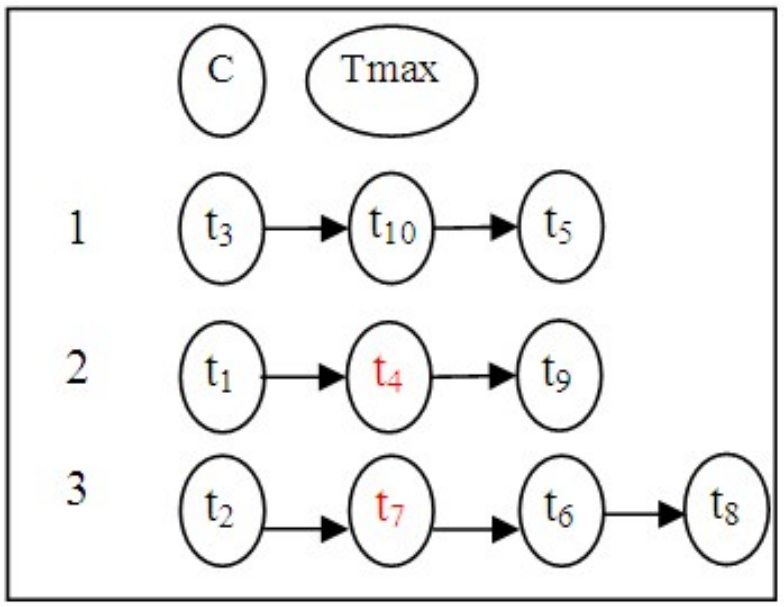

Figure7 Chromosome after using the mutation operator

\section{SIMULATING RESULT AND ANALYSIS}

To test the validity of the algorithm, simulation experiments have been done many times. In the experiments, kinds of data had been used and results are optimum. Here data used in one simulating experiment is as following. Assuming that there are total 20 units of 3 kinds of missiles need to be tested by some missile testing vehicles. The three kind of missiles have separately 6, 7, 7 units, and their testing times are 12, 20, 25 hours. The maximum testing time of all missiles, $T_{\max }$, is supposed as the serial testing time by one vehicle. For the parameters of GA, the scale of the population is taken as 30 . And if the population evolves 50 generations continually, and the optimum solution has no change, then GA ends. Otherwise, the optimum solution is the best solution 
evolved until 300 generations.

The table 1 shows the results when use this algorithm of this paper and the number of testing vehicles, $C$, are $6,7,8,9,10,11,12$, and the results by using serial and parallel method.

TABLE1 THE RESULTS OF THE ALGORITHM

\begin{tabular}{|c|c|c|c|c|c|c|c|c|c|}
\hline & $\begin{array}{c}\text { Serial } \\
\text { Testing }\end{array}$ & \multicolumn{7}{|c|}{ The Algorithm of This Paper } & $\begin{array}{c}\text { Parallel } \\
\text { Testing }\end{array}$ \\
\hline $\begin{array}{c}\text { number of } \\
\text { testing } \\
\text { vehicles }\end{array}$ & 1 & 6 & 7 & 8 & 9 & 10 & 11 & 12 & 20 \\
\hline $\begin{array}{c}\text { total testing } \\
\text { time(hour) }\end{array}$ & 411 & 74 & 57 & 57 & 49 & 45 & 37 & 37 & 25 \\
\hline
\end{tabular}

From table 1, given the number of missiles, types of missiles and the maximum testing time, the algorithm of this paper can get optimum number of missile testing vehicle in different cases. In the experiment, when the number, types, testing times of under-testing missiles are definite, if the maximum limited time given is 57 hours, then 7 missile testing vehicles can meet the request of wartime. Above all, the algorithm has a unique advantage, the corresponding optimum numbers of the testing vehicles using it can be determined simultaneously with different maximum testing times. That is only the number and types of missiles are given, the optimum vehicle within some maximum deadline testing time can be easily confirmed by this algorithm. So this method can optimize the support scheme and save the support cost.

\section{CONCLUSION}

To adapt for the needs of accurate support, we investigated the optimization method based on GA of the missile testing vehicles using for precision-guided missiles. Compared with the traditional algorithms, the method proposed in this paper not only definitely can get optimum value, but also can get a very perfect balance between missile testing cost and testing time. Compared with the traditional method, the method can better meet the future war and can improve the support efficiency

\section{REFERENCES}

[1] Qi Zhengkun, Song Chunxia, Li Ruye. Research on unit package of ammunition support in wartime. Ordnance. 2009,4,25-26

[2] Zhu Xiaodong, Liu Guangyu, Ge Tao. Equipment support of informatization battle. National defense industry press. 2006,57-60

[3] Zhou Ming, Sun Shudong. The principle and application of Genetic Algorithm. National defense industry press, Beijing. 1999,06,4-5.

[4] Wang Xiaoping, Cao Liming. Genetic Algorithm-Its Theory,Application and Software Realization. Xi'an Jiaotong University press, Xi'an.2002,01,12-15

[5] Nirwan Ansari, Edwin Hou. Computional Intelligence for optimization , Qinghua University press, Beijing. 1999,12,49-52 